\title{
MOTIVES AND ALGEBRAIC DE RHAM COHOMOLOGY
}

\author{
MASANORI ASAKURA
}

\begin{abstract}
In this paper, we define a certain Hodge-theoretic structure for an arbitrary variety $X$ over the complex number field by using the theory of mixed Hodge module due to Morihiko Saito. We call it an arithmetic Hodge structure of $X$. It is shown that extension groups of arithmetic Hodge structure do not vanish even for degree $\geq 2$. Moreover, we define higher Abel-Jacobi maps from Bloch's higher Chow groups of $X$ to these extension groups. These maps essentially involve the classical Abel-Jacobi maps by Weil and Griffiths, and Mumford's infinitesimal invariants of 0-cycles on surfaces.
\end{abstract}

\section{INTRODUCTION}

Let $X$ be a nonsingular projective surface over C. D. Mumford was the first to show that the Chow group $\mathrm{CH}_{0}(X)$ of 0 -cycles on $X$ is more complicated than what was earlier believed ( $\mathbb{M}$ ); in fact it can be "enormous". This has led to a difficulty in understanding the precise nature of structure of the Chow group of zero-cycles. His theorem asserts that if the geometric genus of $X$ is not zero, then the kernel $T(X)$ of the Albanese map

$$
A_{0}(X) \longrightarrow \operatorname{Alb}(X)
$$

cannot be finite-dimensional. Namely $A_{0}(X)$ cannot be given a geometric structure such as a complex torus. Here $A_{0}(X)$ denotes the subgroup of 0-cycles of degree 0 .

$\mathrm{S}$. Bloch B1 provided some insight in this situation by conjecturing that $T(X)$ is controlled by the transcendental part of the cohomology $H^{2}(X, \mathbf{Q})_{t r}=H^{2}(X, \mathbf{Q}) / \mathrm{NS}(X)$. This is now called the Bloch conjecture (see Conjecture 4.6 in $\S$ (i). Combining this with the mixed motives as introduced by P. Deligne and A. Beilinson, his conjecture led to a conjectured existence of a filtration $F_{\mathcal{M}}$ on all of the higher Chow groups $\left.\mathrm{CH}^{r}(X, m ; \mathbf{Q})=\mathrm{CH}^{r}(X, m) \otimes \mathbf{Q}(\sqrt{\mathrm{B} 2}]\right)$, called the motivic filtration. (In this paper we will simply write $\mathrm{CH}^{r}(X, m)$ instead of $\mathrm{CH}^{r}(X, m ; \mathbf{Q})$, to be understood as Bloch's higher Chow groups tensored with Q.) This is fortified by the following beautiful conjectural formula for any nonsingular projective variety $X$ :

$$
\operatorname{Gr}_{F_{\mathcal{M}}}^{\nu} \mathrm{CH}^{r}(X, m)=\operatorname{Ext}_{\mathcal{M}}^{\nu}\left(\mathbf{Q}(0), H^{2 r-m-\nu}(X)(r)\right) .
$$

Here $\mathcal{M}$ is the category of mixed motives over $\operatorname{Spec}(\mathbf{C})$ and $\mathbf{Q}(0)$ is the trivial motive. It is proved that the motivic filtration is determined uniquely if it exists (cf. J1, SaS2]). For example, it is conjectured that $F_{\mathcal{M}}^{1} \mathrm{CH}^{r}(X)=\mathrm{CH}^{r}(X)_{\text {hom }}$ (the subgroup of homologically trivial cycles), $F_{\mathcal{M}}^{2} \mathrm{CH}_{0}(X)=T(X)$, and $F_{\mathcal{M}}^{r+1} \mathrm{CH}^{r}(X)=$ 0 . In spite of much effort by many people, nobody has found a suitable definition of $\mathcal{M}$ and $F_{\mathcal{M}}^{\bullet}$ (see [1], Mur1], SaS1]).

Date: ?

1991 Mathematics Subject Classification. ?

The author is supported by JSPS Research Fellowships for Young Scientists. 
On the other hand, the category of the mixed motives $\mathcal{M}$ is considered to possess an exact faithful functor $\mathcal{M} \rightarrow$ MHS (called the realization functor) to the category of graded polarizable $\mathbf{Q}$-mixed Hodge structures. Therefore there should be the map

$$
\operatorname{Gr}_{F_{\mathcal{M}}}^{\nu} \mathrm{CH}^{r}(X) \longrightarrow \operatorname{Ext}_{\mathrm{MHS}}^{\nu}\left(\mathbf{Q}(0), H^{2 r-\nu}(X)(r)\right) .
$$

Unfortunately, the higher extension group $\operatorname{Ext}_{\mathrm{MHS}}^{\nu}(\nu \geq 2)$ always vanishes in the category MHS. This means that $T(X)=\operatorname{Gr}_{F_{\mathcal{M}}}^{2} \mathrm{CH}_{0}(X)$ for a surface $X$ cannot be captured by the extension of mixed Hodge structures.

The purpose of this paper is to define a certain Hodge-theoretic structure for an arbitrary variety $X$ over the complex number field. We call it an arithmetic Hodge structure of $X$. We define the decreasing filtration $F^{\bullet} \mathrm{CH}^{r}(X, m)$ on higher Chow groups and the following map (which we call the higher Abel-Jacobi map):

$$
\rho_{X}^{\nu}: \operatorname{Gr}_{F}^{\nu} \mathrm{CH}^{r}(X, m) \longrightarrow \operatorname{Ext}_{\underline{\mathbf{M}}(\mathbf{C})}^{\nu}\left(\mathbf{Q}(0), H^{2 r-m-\nu}(X)(r)\right) .
$$

Here $\underline{\mathrm{M}}(\mathbf{C})$ denotes the category of arithmetic Hodge structure which possesses the realization functor $r: \underline{\mathrm{M}}(\mathbf{C}) \rightarrow$ MHS. When $\nu=1$, the map $\rho_{X}^{1}$ and the functor $r$ induces the usual Abel-Jacobi maps (cf. §2.5). When $\nu \geq 2$, the higher Abel-Jacobi map $\rho_{X}^{\nu}$ gives a new tool for Hodge theoretic study of algebraic cycles. For example, it defines the second Albanese map

$$
T(X) \longrightarrow \operatorname{Ext}_{\underline{\underline{\mathrm{M}}(\mathbf{C})}}^{2}\left(\mathbf{Q}(0), H^{2 n-2}(X)(n)\right),
$$

for a projective nonsingular variety $X$ of dimension $n$. If it is injective, the Bloch conjecture is true (Theorem 4.9). More generally, we conjecture that $F^{\bullet}$ is discrete, that is, $F^{N}=0$ for $N \gg 0$. If our conjecture holds, our filtration $F^{\bullet}$ gives the motivic filtration (cf. Theorem 1.1 below).

Let us explain arithmetic Hodge structures. Let $X$ be a quasi-projective nonsingular variety over $\mathbf{C}$. Then $X$ is defined by finitely many equations which have finitely many coefficients. By considering the coefficients as parameters of a space $S$, we obtain the smooth family $f: X_{S} \rightarrow S$. Then we get the mixed Hodge module $H^{\bullet}\left(X_{S} / S\right)$ (cf. SaM2). The usual mixed Hodge structure $H^{\bullet}(X, \mathbf{Q})$ appears on the fiber over a point of $S$. The arithmetic Hodge structure is defined to be the inductive limit of the above mixed Hodge modules, $S$ running over the embedding of the function field to $\mathbf{C}$. The most important ingredient of arithmetic Hodge structure is the arithmetic Gauss Manin connection on algebraic de Rham cohomology (see §3.2). Because of this connection, the higher extension group does not necessarily vanish.

Our main result is as follows:

Theorem 1.1. Let $X$ be a nonsingular projective variety over $\mathbf{C}$, and $\mathrm{CH}^{r}(X, m)$ be the higher Chow group tensored with $\mathbf{Q}$. There is a decreasing filtration $F^{\bullet}$ on $\mathrm{CH}^{r}(X, m)$ and a natural map

$$
\rho_{X}^{\nu}: \operatorname{Gr}_{F}^{\nu} \mathrm{CH}^{r}(X, m) \longrightarrow \operatorname{Ext}_{\underline{\mathbf{M}}(\mathbf{C})}^{\nu}\left(\mathbf{Q}(0), H^{2 r-m-\nu}(X)(r)\right),
$$

which has the following properties:

(1) If $F^{r+1} \mathrm{CH}^{r}(X, m)=0$ for arbitrary $X$ and $r$, then $F^{\bullet}$ coincides with the motivic filtration $F_{\mathcal{M}}^{\bullet}$. In particular, the vanishing implies the Bloch conjecture 4.6. 
(2) (a) $F^{0} \mathrm{CH}^{r}(X, m)=\mathrm{CH}^{r}(X, m) \cdot F^{1} \mathrm{CH}^{r}(X)=\mathrm{CH}^{r}(X)_{\text {hom }}$.

(b) $F^{0} \mathrm{CH}^{r}(X, m)=F^{1} \mathrm{CH}^{r}(X, m)$ for $m \geq 1$.

(c) $F^{2} \mathrm{CH}^{r}(X, m)$ is contained in the kernel of the cycle map to the Deligne cohomology group. These coincide if the realization functor $r: \underline{\mathrm{M}}(\mathbf{C}) \rightarrow$ MHS is fully faithful.

(d) $F^{2} \mathrm{CH}_{0}(X)=T(X)$.

(e) $F^{r+1} \mathrm{CH}^{r}(X, m)=F^{r+2} \mathrm{CH}^{r}(X, m)=\cdots$.

(3) There are the following natural maps:

$$
\operatorname{Ext}_{\underline{\underline{\mathbf{M}}}(\mathbf{C})}^{p}\left(\mathbf{Q}(0), H^{q}(X)(r)\right) \longrightarrow \Xi_{X}^{r-p, q-r+p}(p) \longrightarrow \Lambda_{X}^{r-p, q-r+p}(p),
$$

where $\Xi_{X}^{p, q}(r)$ (resp. $\Lambda_{X}^{p, q}(r)$ ) is defined as the cohomology of the following complex (see Definition 3.2 and Definition 3.5):

$$
\begin{gathered}
F^{p+1} H_{d R}^{p+q}(X / \mathbf{C}) \otimes \Omega_{\mathbf{C} / \overline{\mathbf{Q}}}^{r-1} \stackrel{\nabla}{\rightarrow} F^{p} H_{d R}^{p+q}(X / \mathbf{C}) \otimes \Omega_{\mathbf{C} / \overline{\mathbf{Q}}}^{r} \stackrel{\nabla}{\rightarrow} F^{p-1} H_{d R}^{p+q}(X / \mathbf{C}) \otimes \Omega_{\mathbf{C} / \overline{\mathbf{Q}}}^{r+1}, \\
\left(\text { resp. } H^{q-1}\left(\Omega_{X / \mathbf{C}}^{p+1}\right) \otimes \Omega_{\mathbf{C} / \overline{\mathbf{Q}}}^{r-1} \stackrel{\bar{\nabla}}{\rightarrow} H^{q}\left(\Omega_{X / \mathbf{C}}^{p}\right) \otimes \Omega_{\mathbf{C} / \overline{\mathbf{Q}}}^{r} \stackrel{\bar{\nabla}}{\rightarrow} H^{q+1}\left(\Omega_{X / \mathbf{C}}^{p-1}\right) \otimes \Omega_{\mathbf{C} / \overline{\mathbf{Q}}}^{r+1} .\right.
\end{gathered}
$$

In particular, the map (1.1.2) induces homomorphisms

$$
\xi_{X}^{\nu}: \operatorname{Gr}_{F}^{\nu} \mathrm{CH}^{r}(X, m) \longrightarrow \Xi_{X}^{r-\nu, r-m}(\nu)
$$

and

$$
\delta_{X}^{\nu}: \mathrm{Gr}_{F}^{\nu} \mathrm{CH}^{r}(X, m) \longrightarrow \Lambda_{X}^{r-\nu, r-m}(\nu) .
$$

In general, these maps have non-zero images, even when $\nu \geq 2$, (which follows, for example, from Theorem 4.3).

(4) Assume that $X$ is defined over a number field $k$, and $X=X_{k} \otimes_{k}$ C. If $F^{\operatorname{dim} X+1} \mathrm{CH}_{0}(X)=0$, then $F^{2} \mathrm{CH}_{0}\left(X_{k}\right)=0$ and the rank of $\mathrm{CH}_{0}\left(X_{k}\right)$ is finite.

We call the above map (1.1.2) the $\nu$-th higher Abel-Jacobi map. Also, we remark that philosophically speaking, the fact that $F^{0} \mathrm{CH}^{r}(X, m)=F^{1} \mathrm{CH}^{r}(X, m)$ for $m \geq 1$ should not come as a surprise. Indeed, for $m \geq 1$ and by a standard weight argument, the cycle class map from $\mathrm{CH}^{r}(X, m)$ to ordinary Betti cohomology is zero. Thus $\mathrm{CH}^{r}(X, m)$ is already the kernel of a "regulator" map.

By way of acknowledgement, a similar idea of arithmetic Hodge structures was obtained by M. Green and P. Griffiths ([G2]). They defined the arithmetic Hodge structures independently of the author円. The terminology "arithmetic Hodge structure" was introduced by them. It is appropriate to adopt their terminology in this paper. However, our motivation for the above idea comes from the the work of Shuji Saito; that is, the higher normal functions $([\mathrm{AS}])$. He defined the relative filtration $F_{S}^{\bullet} \mathrm{CH}^{\bullet}(X)$ and likewise $F_{S}^{\bullet} H_{D}^{\bullet}(X, \mathbf{Q}(\bullet))$ for a smooth projective family $X \rightarrow S$, and defined higher normal functions

$$
\mathrm{Gr}_{F_{S}}^{\nu} \mathrm{CH}^{r}(X) \longrightarrow \Gamma\left(S, D J_{X / S}^{r, \nu}\right) .
$$

It was surprising for us that the above map has non-trivial image.

This paper is organized as follows.

In $\S 2$, we review Morihiko Saito's theory of mixed Hodge modules. Here we omit the definition, because what we need is not the precise definition but rather the

\footnotetext{
${ }^{1}$ There is a slight difference between their definition and ours. In their definition, the datum of the Q-structure does not appear. Therefore the category of their arithmetic Hodge structures does not form an abelian category, but only an exact category.
} 
formalism. In $\S 3$, we introduce the notion of arithmetic Hodge structure. Moreover we define the spaces of Mumford's infinitesimal invariants, and construct the natural maps from extension groups in $\underline{\mathrm{M}}(\mathbf{C})$ to those (Proposition 3.6). Finally in $\S 4$, we construct the higher Abel-Jacobi maps, and study algebraic cycles, in particular, the Bloch conjecture.

\section{Acknowledgment}

We are especially indebted to Shuji Saito for his many helpful suggestions. It is my great pleasure to have had the opportunity to work together with him.

We thank Shin-ichi Mochizuki, who kindly provided a proof for the existence of the curve which appears in Theorem 1.5, and Morihiko Saito, who has read through this paper carefully and has come up with many helpful comments.

We also thank Sampei Usui and Takeshi Saito for fruitful discussions and constant encouragement.

Special thanks are due to the two referees who have read through the earlier versions of this paper pointing out many inaccuracies and suggesting numerous improvements.

At last but not least, we would like to thank Noriko Yui and James D. Lewis for their help for improving English and mathematical presentations putting the paper into a presentable form to the reader. Without their generous help and patience, this paper has never reached this final form.

It is great pleasure to thank the organizers of the Banff Conference on "Arithmetic and Geometry of Algebraic Cycles" for the excellent conference.

\section{Notation and Conventions}

1. A variety means a quasi-projective algebraic variety over a field. We mainly work with algebraically closed fields of characteristic 0 (e.g. C, $\overline{\mathbf{Q}}$ ).

2. For a variety $X$ over $\mathbf{C}, X^{a n}$ denotes the associated analytic space: $X^{a n}=$ $X(\mathbf{C})$.

3. We always ignore torsion. We assume that all abelian groups (e.g. $\mathrm{CH}^{r}(X, m)$ above) are tensored with $\mathbf{Q}$.

4. In this paper, we fix an embedding $\overline{\mathbf{Q}} \hookrightarrow \mathbf{C}$.

\section{Morihiko Saito's formalism of miXed Hodge modules}

In this section, we review the theory of mixed Hodge modules.

2.1. Perverse sheaf. A perverse sheaf is not a "sheaf" in the usual sense, but rather a complex of sheaves with algebraically constructible cohomology. In many ways it behaves like a sheaf ( $\mathrm{BBD})$.

Let $D_{c}^{b}\left(\mathbf{Q}_{X}\right)$ be the derived category of bounded complexes of $\mathbf{Q}_{X^{a n} \text {-sheaves }}$ with algebraically constructible cohomology for a complex algebraic variety $X$. The perverse $t$-structure $\left(D^{\geq 0}, D^{\leq 0}\right)$ of $D_{c}^{b}\left(\mathbf{Q}_{X}\right)$ is the pair of additive full subcategories defined as follows:

(1) $K_{\mathbf{Q}}^{\bullet} \in D^{\leq 0} \Longleftrightarrow \operatorname{dimSupp} H^{i}\left(K_{\mathbf{Q}}^{\bullet} \leq-i \quad(\forall i)\right.$

(2) $K_{\mathbf{Q}}^{\bullet} \in D^{\geq 0} \Longleftrightarrow \mathbb{D}\left(K_{\mathbf{Q}}^{\bullet}\right) \in D^{\leq 0} \quad(\forall i)$, where $\mathbb{D}$ is the Verdier dual functor on $D_{c}^{b}\left(\mathbf{Q}_{X}\right)$.

Here we define the dimension of empty set to be $-\infty$. We call an object of $D^{\geq 0} \cap D^{\leq 0}$ a perverse sheaf, and denote its category by $\operatorname{Perv}\left(\mathbf{Q}_{X}\right)$. 
The category of perverse sheaves is abelian. There are the truncation functors ${ }^{p} \tau_{\leq 0}: D_{c}^{b}\left(\mathbf{Q}_{X}\right) \rightarrow D^{\leq 0}$ and ${ }^{p} \tau_{\geq 0}: D_{c}^{b}\left(\mathbf{Q}_{X}\right) \rightarrow D^{\geq 0}$, which are characterized as the right and left adjoint of $D^{\leq 0} \hookrightarrow D_{c}^{b}\left(\mathbf{Q}_{X}\right)$ and $D^{\geq 0} \hookrightarrow D_{c}^{b}\left(\mathbf{Q}_{X}\right)$ respectively. We put ${ }^{p} H^{0}:={ }^{p} \tau_{\leq 0}{ }^{p} \tau_{\geq 0}\left(\simeq{ }^{p} \tau_{\geq 0}{ }^{p} \tau_{\leq 0}\right)$, and call it the perverse cohomology functor.

There are the standard functors on $D_{c}^{b}\left(\mathbf{Q}_{X}\right)$ :

$$
f_{*}, f_{!}, f^{*}, f^{!}, \mathbb{D}, \psi_{g}, \phi_{g}, \otimes, \underline{\text { Hom. }} .
$$

We write ${ }^{p} H^{0} f_{*}(-)[n]$ by ${ }^{p} R^{n} f_{*}$ and so on.

The simplest example of perverse sheaf is a local system $L[\operatorname{dim} X]$ shifted by $\operatorname{dim} X$ when $X$ is nonsingular. The category of local systems is not appropriate for the treatment of homological algebra. Indeed, it is not closed under the above standard functors. The category of constructible sheaves is closed under those, but the one of perverse sheaves has more advantages. Those properties of perverse sheaves are essentially used to construct the theory of mixed Hodge modules.

2.2. Regular holonomic $D$-modules. Next we recall holonomic $D$-modules? regular singularities.

Let $X$ be a nonsingular variety over a field $k$ of characteristic zero.

Let $M$ be an algebraic $D_{X}$-module which is quasi-coherent over $\mathcal{O}_{X}$. We denote $F_{i} D_{X} \subset D_{X}$ the differential order filtration, that is, it is generated by differential operators $\sum_{|I| \leq i} f_{I}(x) \partial^{I}$. An increasing filtration $F_{\bullet} M$ of coherent $\mathcal{O}_{X}$-submodules is called a good filtration if

1. $\cup_{i} F_{i} M=M, F_{-k} M=0$ for some integer $k \gg 0$,

2. $F_{i} D_{X} \cdot F_{j} M \subset F_{i+j} M$ for $\forall i, j$,

3. $\operatorname{gr}^{F} M:=\oplus_{i} \operatorname{gr}_{i}^{F} M$ is coherent over $\operatorname{gr}^{F} D_{X}$.

Any coherent $D_{X}$-module has at least one good filtration. Note that $\operatorname{gr}^{F} D_{X}$ is isomorphic to $\mathcal{O}_{T^{*} X}$ the structure sheaf of the cotangent bundle $T^{*} X$ over $X$. The support of $\operatorname{gr}^{F} M$ in $T^{*} X$ is called the characteristic variety of $M$, which does not depend on the choice of a good filtration.

In general, the dimension of the characteristic variety of a coherent $D_{X}$-module is larger than or equal to $\operatorname{dim} X$ (Bernstein's inequality). If the equality holds, we call the coherent $D_{X}$-module holonomic.

Let us recall the regularity of holonomic $D$-module ${ }^{\text {f }}$. When $\operatorname{dim} X=1$, a holonomic $D_{X}$-module $M$ has regular singularities if and only if there is a dense open set $U \subset X$ such that $\left.M\right|_{U}$ is a connection with regular singularities, that is, there is a locally free sheaf $\bar{M}$ of finite rank over $\mathcal{O}_{\bar{U}}(\bar{U}$ is the smooth completion of $U)$ and $\log$ connection $\bar{\nabla}: \bar{M} \rightarrow \Omega \frac{1}{U / k}(\log D) \otimes \bar{M}(D:=\bar{U}-U)$ such that $\left.(\bar{M}, \bar{\nabla})\right|_{U} \simeq\left(\left.M\right|_{U}, \nabla\right)$. For $X$ of arbitrary dimension, a holonomic $D_{X}$-module $M$ has regular singularities if and only if so does $H^{0}\left(i_{C}^{*} M\right)$ for any $i_{C}: C \rightarrow X$ with $C$ a nonsingular curve over $k$, where $i_{C}^{*}$ is the derived inverse image functor of $D$-modules.

The following theorem is due to Kashiwara and Mebkhout:

\footnotetext{
${ }^{2}$ We use left $D$-modules, though M.Saito prefers right $D$-modules to it.

${ }^{3}$ There are several ways to define regularities of holonomic $D$-modules. We follow the text of Z.Mebkhout $\mathrm{Me}$ p.163 Proposition 5.4.2.
} 
Theorem 2.1 (Riemann-Hilbert correspondence). Let $X$ be a nonsingular variety over $\mathbf{C}$. Then the de Rham functor $\mathrm{DR}:=\Omega_{X^{a n}}^{\bullet} \otimes(-)[\operatorname{dim} X]$ induces the equivalence from the category of regular holonomic D-modules on $X$ to the one of perverse sheaves with $\mathbf{C}$-coefficient:

$$
\text { DR : } \operatorname{Mod}_{r h}(X) \stackrel{\sim}{\longrightarrow} \operatorname{Perv}\left(\mathbf{C}_{X}\right) .
$$

The above equivalence also induces the equivalence between local systems and locally free $\mathcal{O}_{X}$-sheaves with integrable connection with regular singularities (D3]).

There are the standard operations as in 2.2.1 on the derived category of bounded complexes of regular holonomic $D$-modules. Those operations are compatible under the Riemann-Hilbert correspondence.

Again, let $X$ be a nonsingular variety over any field of characteristic zero. $\mathrm{MF}_{r h}(X)$ denotes the category of filtered regular holonomic $D_{X}$-modules $(M, F)$, where $M$ is a regular holonomic $D_{X}$-module, and $F$ is a good filtration. Moreover we denote by $\operatorname{MFW}_{r h}(X)$ the category of filtered regular holonomic $D_{X}$-modules with weight filtration $(M, F, W)$, where $W$ is a finite increasing filtration of $D_{X^{-}}$ submodules (=the weight filtration). These are not abelian, but exact categories such that the kernel and cokernel objects exist for any morphisms. A complex in $\operatorname{MF}_{r h}(X)\left(\right.$ resp. $\operatorname{MFW}_{r h}(X)$ )

$$
\begin{gathered}
\left(M^{\prime}, F\right) \longrightarrow(M, F) \longrightarrow\left(M^{\prime \prime}, F\right) \\
\left(\operatorname{resp} .\left(M^{\prime}, F, W\right) \longrightarrow(M, F, W) \longrightarrow\left(M^{\prime \prime}, F, W\right)\right)
\end{gathered}
$$

is exact if and only if

$$
\begin{gathered}
\operatorname{gr}^{F} M^{\prime} \longrightarrow \operatorname{gr}^{F} M \longrightarrow \operatorname{gr}^{F} M^{\prime \prime} \\
\left(\text { resp. } \operatorname{gr}^{F} \operatorname{gr}^{W} M^{\prime} \longrightarrow \operatorname{gr}^{F} \operatorname{gr}^{W} M \longrightarrow \operatorname{gr}^{F} \operatorname{gr}^{W} M^{\prime \prime}\right)
\end{gathered}
$$

is an exact sequence of sheaves of $\mathcal{O}_{X}$-modules.

2.3. Mixed Hodge modules. Morihoko Saito defined mixed Hodge modules, and proved the expected properties.

What is a mixed Hodge module? Roughly speaking, it is a $\mathbf{Q}$-coefficient perverse sheaf with mixed Hodge structure. Its notion contains admissible variation of mixed Hodge structure.

First we recall it:

Definition 2.2. Let $X$ be a nonsingular variety over $\mathbf{C}$. Then an admissible variation of mixed Hodge structures on $X$ is defined to be the data $\left(H_{\mathbf{Q}}, H_{\mathcal{O}}, W_{\bullet}, F^{\bullet}, \nabla, i\right)$ where:

- $H_{\mathbf{Q}}$ is a local system of finite dimensional $\mathbf{Q}$-vector spaces on $X^{a n}$,

- $H_{\mathcal{O}}$ is a locally free (Zariski) sheaf of $\mathcal{O}_{X}$-module of the same rank as $H_{\mathbf{Q}}$,

- $W_{\bullet}$ is a finite increasing filtration of $H_{\mathbf{Q}}$, called the weight filtration,

- $F^{\bullet}$ is a finite decreasing filtration on $H_{\mathcal{O}}$ by locally free $\mathcal{O}_{X}$-submodules, called the Hodge filtration,

- $\nabla: H_{\mathcal{O}} \rightarrow H_{\mathcal{O}} \underset{\mathcal{O}_{X}}{\otimes} \Omega_{X / \mathbf{C}}^{1}$ an integrable connection (called the (algebraic) Gauss-Manin connection),

- $i: H_{\mathbf{Q}} \otimes \mathbf{C} \stackrel{\sim}{\longrightarrow} \operatorname{ker} \nabla^{a n}$ (called the comparison isomorphism), or equivalently, $i$ induces an isomorphism $H_{\mathbf{Q}} \otimes \mathcal{O}_{X}^{a n} \stackrel{\sim}{\rightarrow} H_{\mathcal{O}}^{a n}$.

and these satisfy: 
(1) For all points $s \in X^{a n}$, the fiber $H_{\mathbf{Q}, s} \stackrel{i}{\hookrightarrow} H_{\mathcal{O}} \otimes \mathbf{C}(s)$ with the induced filtrations $W_{\bullet, s}$ on $H_{\mathbf{Q}, s}$ and $F_{s}^{\bullet}$ on $H_{\mathcal{O}} \otimes \mathbf{C}(s)$ defines a mixed Hodge structure.

(2) (Griffiths transversality) $W_{\bullet}$ and $F^{\bullet}$ satisfy the following:

$$
\nabla\left(W_{\ell}\right) \subset W_{\ell} \otimes \Omega_{X / \mathbf{C}}^{1}, \quad \nabla\left(F^{p}\right) \subset F^{p-1} \otimes \Omega_{X / \mathbf{C}}^{1} \quad \text { for } \forall \ell, p .
$$

(3) (polarizability) For each $\ell$, there are $\mathbf{Q}$-bilinear form $Q: \mathrm{Gr}_{\ell}^{W}\left(H_{\mathbf{Q}}\right) \otimes \mathrm{Gr}_{\ell}^{W}\left(H_{\mathbf{Q}}\right) \rightarrow$ $\mathbf{Q}$ and $\mathcal{O}_{X}$-bilinear form $Q_{X}: \operatorname{Gr}_{\ell}^{W}\left(H_{\mathcal{O}}\right) \otimes \operatorname{Gr}_{\ell}^{W}\left(H_{\mathcal{O}}\right) \rightarrow \mathcal{O}_{X}$ satisfying:

(a) $Q$ and $Q_{X}$ are compatible under the comparison isomorphism $i$.

(b) $Q$ defines a polarization form on the $\mathbf{Q}$-Hodge structure $\left(\mathrm{Gr}_{\ell}^{W}\left(H_{\mathbf{Q}, s}\right), F_{s}^{\bullet}\right)$ for all $s \in X^{a n}$.

(c) $Q_{X}(\nabla(x), y)+Q_{X}(x, \nabla(y))=d Q_{X}(x, y)$ for any local sections $x, y \in H_{\mathcal{O}}$.

(4) (admissibility) When the data $\left(H_{\mathbf{Q}}, H_{\mathcal{O}}, W_{\bullet}, F^{\bullet}, \nabla, i\right)$ is pulled back to a nonsingular complex algebraic curve $C$, it satisfies the admissibility ([K] 1.9):

(a) Any local monodromy around $\bar{C}-C$ is quasi-unipotent. Here $\bar{C}$ denotes the smooth completion of $C$.

(b) The logarithm $N$ of the unipotent part of the local monodromy admits a weight filtration relative to $W_{\bullet}($ SZ $\$$ [2).

(c) The Hodge filtration $F^{\bullet}$ can be extended to a locally free subsheaf $\bar{F}^{\bullet}$ of the Deligne's canonical extension $\bar{H}_{C}$ such that $\operatorname{Gr}_{\bar{F}}^{\bullet} \operatorname{Gr}_{\bullet}^{\bar{W}}\left(\bar{H}_{C}\right)$ is locally free.

We denote the category of admissible variations of mixed Hodge structures on $X$ by $\operatorname{VMHS}(X)$.

In order to define mixed Hodge module, we replace $H_{\mathbf{Q}}$ by perverse sheaf, $\left(H_{\mathcal{O}}, F^{\bullet}, \nabla\right)$ by filtered regular holonomic $D_{X}$-module, and $i$ by the RiemannHilbert correspondence.

More precisely, let $\operatorname{PervW}\left(\mathbf{Q}_{X}\right)$ be the category of perverse sheaves $\left(K_{\mathbf{Q}}^{\bullet}, W_{\mathbf{Q}}\right)$ with finite increasing filtration $W_{\mathbf{Q}}$ of sub perverse sheaves (=the weight filtration). There is a natural functor from $\mathrm{MFW}_{r h}(X)$ to $\operatorname{PervW}\left(\mathbf{C}_{X}\right)$, which maps $(M, F, W)$ to $(\operatorname{DR}(M), \operatorname{DR}(W))$. Denote the fiber product $\operatorname{PervW}\left(\mathbf{Q}_{X}\right) \times \operatorname{PervW}\left(\mathbf{C}_{X}\right)$ $\operatorname{MFW}_{r h}(X)$ by $\operatorname{MFW}_{r h}(X, \mathbf{Q})$, that is, it consists of the objects $\left(K_{\mathbf{Q}}^{\bullet}, W_{\mathbf{Q}}, M, F, W, i\right)$ where $i:\left(K_{\mathbf{Q}}^{\bullet} \otimes \mathbf{C}, W_{\mathbf{Q}} \otimes \mathbf{C}\right) \simeq \mathrm{DR}(M, W)$ is an isomorphism of filtered objects (cf. Theorem 2.1).

Theorem 2.3 (Morihiko Saito SaM1, SaM2]). There is an abelian full subcategory

$$
\operatorname{MHM}(X) \subset \operatorname{MFW}_{r h}(X, \mathbf{Q})
$$

for each nonsingular complex algebraic variety $X$, which satisfies the following properties. We call an object of $\operatorname{MHM}(X)$ a mixed Hodge module on $X . W$ (resp. F ) is called the weight filtration (resp. Hodge filtration).

(1) The forgetful functor rat: $\operatorname{MHM}(X) \rightarrow \operatorname{Perv}\left(\mathbf{Q}_{X}\right)$ is exact and faithful.

(2) Any morphisms of mixed Hodge modules are strict with respect to Hodge and weight filtration.

(3) Any pure weight full subcategory of $\operatorname{MHM}(X)$ (called polarizable Hodge modules) is semi-simple.

(4) There are the standard operations on $D^{b}(\operatorname{MHM}(X))$ the derived category of bounded complex of mixed Hodge modules:

$$
f_{*}, f_{!}, f^{*}, f^{!}, \mathbb{D}, \psi_{g}, \phi_{g, 1}, \otimes, \underline{\text { Hom. }} .
$$


Those functors satisfies the adjointness, projection formulas, and are compatible with the ones on perverse sheaves under the forgetful functor rat.

(5) Let $\operatorname{MHM}(X)_{\mathrm{sm}}$ be the full subcategory of $\operatorname{MHM}(X)$ whose object consists of those whose underlying perverse sheaf is smooth (i.e. local system). Then there is the equivalence between the category of admissible variations of mixed Hodge structure ( Definition 2.2) and $\operatorname{MHM}(X)_{\mathrm{sm}}$ :

$$
\operatorname{VMHS}(X) \stackrel{\sim}{\longrightarrow} \operatorname{MHM}(X)_{\mathrm{sm}} .
$$

Here an admissible variation of mixed Hodge structure $\left(H_{\mathbf{Q}}, H_{\mathcal{O}}, W_{\bullet}, F^{\bullet}, \nabla, i\right)$ corresponds to the mixed Hodge module $\left(H_{\mathbf{Q}}[\operatorname{dim} X], W_{\bullet}-\operatorname{dim} X, H_{\mathcal{O}}, F_{\bullet}\right)$, where $F_{p}:=F^{-p}$. An admissible variation of Hodge structure of pure weight $n$ corresponds to the Hodge module of weight $n+\operatorname{dim} X$. In particular, $\mathrm{MHM}(\operatorname{SpecC})$ is isomorphic to the category of graded polarizable $\mathbf{Q}$-mixed Hodge structures.

(6) (decomposition theorem) Let $f: X \rightarrow Y$ be a proper morphism between nonsingular varieties over $\mathbf{C}$. Then there is a noncanonical isomorphism

$$
f_{*} M \simeq \underset{k}{\oplus} H^{k} f_{*} M[-k]
$$

in the derived category $D^{b}(\operatorname{MHM}(Y))$ for any pure weight Hodge module $M$.

(7) If $H$ is a mixed Hodge module of weight $\leq n$ (resp. $\geq n)$, then $H^{k} f_{!}(H)$ and $H^{k} f^{*}(H)\left(\right.$ resp. $H^{k} f_{*}(H), H^{k} f^{!}(H)$, resp. $\left.\mathbb{D} H\right)$ are of weight $\leq k+n$ (resp. $\geq k+n$, resp. $\leq-n)$.

Morihiko Saito firstly defined the category of polarizable Hodge modules using vanishing cycle functor, and proved the stability of direct images of projective morphism, Verdier dual and so on, which is the main result in SaM1. Next he defined mixed Hodge modules as a successive extension of Hodge modules satisfying some stability conditions ([SaM2 $)$. The most important property is the existence of standard functors (loc.cit. Theorem 4.3. etc). However, the precise construction of the category of mixed Hodge modules is long and complicated. Moreover it is quite difficult to write it down explicitly.

Definition 2.4. $\mathbf{Q}_{X}(r)[\operatorname{dim} X]$ denotes the Tate Hodge module

$$
\left(\mathbf{Q}_{X}(r)[\operatorname{dim} X], W_{\bullet}, \mathcal{O}_{X}, F_{\bullet}\right)
$$

with $\operatorname{Gr}_{\operatorname{dim} X-2 r}^{W}\left(\mathbf{Q}_{X}(r)[\operatorname{dim} X]\right)=\mathbf{Q}_{X}(r)[\operatorname{dim} X]$ and $\operatorname{Gr}_{r}^{F}\left(\mathcal{O}_{X}\right)=\mathcal{O}_{X}$, (which is actually a mixed Hodge module of pure weight $\operatorname{dim} X-2 r$ due to Theorem 2.3 (5).)

We write the Tate Hodge module shifted by $-\operatorname{dim} X$ by $\mathbf{Q}_{X}(r)$ :

$$
\mathbf{Q}_{X}(r):=\left(\mathbf{Q}_{X}(r)[\operatorname{dim} X]\right)[-\operatorname{dim} X] \in D^{b}(\operatorname{MHM}(X)) .
$$

2.4. Mixed Hodge modules over a field of characteristic 0. Morihiko Saito has already pointed out that there are several modifications of mixed Hodge modules. Here we recall one of those.

Definition 2.5. Let $k \subset \mathbf{C}$ be a subfield. For a nonsingular variety $X_{k}$ over $k$, we define $\operatorname{MHM}\left(X_{k}\right)$ to be the full subcategory of the fiber product

$$
\operatorname{MFW}_{r h}\left(X_{k}\right) \times_{\mathrm{MFW}_{r h}\left(X_{\mathbf{C}}\right)} \operatorname{MHM}\left(X_{\mathbf{C}}\right) \quad\left(X_{\mathbf{C}}:=X_{k} \otimes \mathbf{C}\right)
$$

such that the polarization on each weight quotient is defined over $k$. 
Since all operations such as 2.2.1) can be also defined on $\operatorname{MFW}_{r h}\left(X_{k}\right)$, we have those induced on $\operatorname{MHM}\left(X_{k}\right)$. Each property of those operations can be reduced to those of $\operatorname{MHM}\left(X_{\mathbf{C}}\right)$. In particular, Theorem 2.3 also holds, if we replace $\operatorname{MHM}\left(X_{\mathbf{C}}\right)$ by $\operatorname{MHM}\left(X_{k}\right)$, and $\operatorname{VMHS}\left(X_{\mathbf{C}}\right)$ by the full subcategory of $\operatorname{MFW}_{r h}\left(X_{k}\right)_{\mathrm{sm}} \times_{\mathrm{MFW}_{r h}\left(X_{\mathbf{C}}\right)_{\mathrm{sm}}} \operatorname{VMHS}\left(X_{\mathbf{C}}\right)$ such that the polarization on each weight quotient is defined over $k$. $\left(\mathrm{MFW}_{r h}\left(X_{k}\right)_{\mathrm{sm}}\right.$ denotes the full subcategory of $\mathrm{MFW}_{r h}\left(X_{k}\right)$ generated by objects whose underlying $D$-module is a locally free $\mathcal{O}_{X}$-module of finite rank.) For more details, see his exposition (SaM3 (1.8) (ii)).

Corollary 2.6. Let $f: X \rightarrow Y$ be a proper morphism between nonsingular varieties over $k$. Then there is the Leray spectral sequence

$$
E_{2}^{p q}=\operatorname{Ext}_{\mathrm{MHM}(Y)}^{p}\left(\mathbf{Q}_{Y}(0), H^{q} f_{*} \mathbf{Q}_{X}(r)\right) \Longrightarrow \operatorname{Ext}_{\operatorname{MHM}(X)}^{p+q}\left(\mathbf{Q}_{X}(0), \mathbf{Q}_{X}(r)\right),
$$

which degenerates at $E_{2}$ terms.

Proof. The existence of the spectral sequence (2.2.2) follows from the existence of the standard functors (Theorem 2.3 (画) and the fact $f^{*} \mathbf{Q}_{Y}(r)=\mathbf{Q}_{X}(r)$. The $E_{2}$-degeneration follows from the decomposition theorem (Theorem 2.3 (6)).

Corollary 2.7 (SaM3 (8.3)). Let $X$ be a nonsingular variety over $k$. Then there is a cycle map from the higher Chow group to the extension group of mixed Hodge modules:

$$
\mathrm{CH}^{r}(X, m) \longrightarrow \operatorname{Ext}_{\operatorname{MHM}(X)}^{2 r-m}\left(\mathbf{Q}_{X}(0), \mathbf{Q}_{X}(r)\right)
$$

Proof. (Here we recall the construction of the cycle map 2.2.3) for the convenience of the reader.)

Let $Y$ be a nonsingular variety over $k$ of dimension $d_{Y}$, and $i_{Z}: Z \hookrightarrow Y$ a closed subscheme (not necessarily smooth nor irreducible) of pure dimension $d_{Z}$. Let $j_{Z}: U \hookrightarrow Y$ be the complement of $Z$. We define $i_{Z *} i_{Z} \mathbf{Q}_{Y}(r)=$ $\operatorname{Cone}\left(\mathbf{Q}_{Y}(r) \rightarrow j_{Z *} j_{Z}^{*} \mathbf{Q}_{Y}(r)\right)[-1]$, and $i_{Z *} \mathbf{Q}_{Z}(r)=\operatorname{Cone}\left(j_{Z !} j_{Z}^{*} \mathbf{Q}_{Y}(r) \rightarrow \mathbf{Q}_{Y}(r)\right)$, which are complexes of mixed Hodge modules on $Y$ by Theorem 2.3 (4). Note that $\operatorname{rat}\left(i_{Z *} \mathbf{Q}_{Z}(r)\right)=i_{Z *} \mathbf{Q}_{Z^{a n}}$ and $\operatorname{rat}\left(i_{Z *} i_{Z}^{!} \mathbf{Q}_{Y}(r)\right)=i_{Z *} i_{Z} \mathbf{Q}_{Y^{a n}}$.

Let $Z_{k}$ be an irreducible component of $Z$, and $z=\sum_{k} n_{k} Z_{k}$ be a cycle on $Y$ with $n_{k} \in \mathbf{Q}$. First we construct the natural morphism

$$
c_{z}: i_{Z *} \mathbf{Q}_{Z}(0) \longrightarrow \mathbf{Q}_{Y}\left(d_{Y}-d_{Z}\right)\left[2 d_{Y}-2 d_{Z}\right]
$$

associated to the cycle $z$ in the derived category $D^{b}(\operatorname{MHM}(Y))$ as follows. Let $h_{k}$ : $\widetilde{Z}_{k} \rightarrow Z_{k}$ be a resolution of singularity, and $h: \amalg \widetilde{Z}_{k} \rightarrow Y$ the composition with the inclusion $i_{Z}$. There are the pull-back morphisms $\alpha: i_{Z *} \mathbf{Q}_{Z}(0) \rightarrow i_{Z *} h_{k *} \mathbf{Q}_{\widetilde{Z}_{k}}(0)^{\oplus}=$ $h_{*} \mathbf{Q}_{\widetilde{Z}_{k}}(0)^{\oplus}$ and $\beta: \mathbf{Q}_{Y}(0) \rightarrow h_{*} \mathbf{Q}_{\widetilde{Z}_{k}}(0)^{\oplus}$. Applying the Verdier dual $\mathbb{D}$ on the latter morphism $\beta$, we have $h_{*} \mathbf{Q}_{\widetilde{Z}_{k}}\left(d_{Z}\right)^{\oplus}\left[2 d_{Z}\right] \rightarrow \mathbf{Q}_{Y}\left(d_{Y}\right)\left[2 d_{Y}\right]$ because $Y$ and $Z_{k}$ are nonsingular. Composing this with $\oplus n_{k}: h_{*} \mathbf{Q}_{\widetilde{Z}_{k}}(0)^{\oplus} \rightarrow h_{*} \mathbf{Q}_{\widetilde{Z}_{k}}(0)^{\oplus}$ and the morphism $\alpha$, we have 2.2.4). It is a routine work to show its independency of the choice of resolutions of $Z_{k}$.

Put $Y=X$, and compose the morphism (2.2.4) with $\mathbf{Q}_{X}(0) \rightarrow i_{Z *} \mathbf{Q}_{Z}(0)$. Then we have the cycle map (2.2.3) for $m=0$. 
Next we construct the cycle map 2.2.3 for $m \geq 1$. We write

$$
\Delta^{m}=\operatorname{Spec} k\left[t_{0}, \cdots, t_{m}\right] /\left(\sum_{i=0}^{m} t_{i}-1\right)
$$

and its faces by

$$
\Delta_{I}: t_{i_{0}}=\cdots=t_{i_{k}}=0
$$

for a subset $I=\left\{i_{0}, \cdots, i_{k}\right\} \subset\{0, \cdots, m\}$. Put $Y=X \times \Delta^{m}$, and $i: X \times\left(\cup_{i} \Delta_{i}\right) \hookrightarrow$ $Y$ the closed immersion and $j: X \times U \hookrightarrow Y$ its complement. Let $z=\sum n_{k} Z_{k}$ be a cycle on $Y$ (where $Z_{k}$ are reduced and irreducible subvarieties of dimension $d_{Y}-r$ ) which intersects with all faces $X \times \Delta_{I}$ properly, and

$$
z \cdot\left[X \times \Delta_{i}\right]=0 \text { for } 0 \leq \forall i \leq m \text {. }
$$

Put $Z=\cup_{k} Z_{k}$ the support of $z$.

We first claim:

Lemma 2.8. The morphism $c_{z}$ 2.2.4 is uniquely lifted to the morphism

$$
i_{Z *} \mathbf{Q}_{Z}(0) \longrightarrow j_{!} j^{*} \mathbf{Q}_{Y}(r)[2 r] .
$$

In fact, using the distinguished triangle

$$
j_{!} j^{*} \mathbf{Q}_{Y}(r) \longrightarrow \mathbf{Q}_{Y}(r) \longrightarrow i_{*} \mathbf{Q}_{X \times\left(\cup_{i} \Delta_{i}\right)}(r) \stackrel{+1}{\longrightarrow},
$$

it follows from

$$
\operatorname{Ext}_{\mathrm{MHM}(Y)}^{\nu}\left(i_{Z *} \mathbf{Q}_{Z}(0), i_{*} \mathbf{Q}_{X \times\left(\cup_{i} \Delta_{i}\right)}(r)\right)=0 \quad \text { for } \forall \nu<2 r,
$$

and

$$
\text { the composition of } c_{z} \text { with } \mathbf{Q}_{Y}(r) \longrightarrow i_{*} \mathbf{Q}_{X \times\left(\cup_{i} \Delta_{i}\right)}(r) \text { is zero. }
$$

Since $i_{*} \mathbf{Q}_{X \times\left(\cup_{i} \Delta_{i}\right)}(r)$ is isomorphic to

$$
\bigoplus_{i=0}^{m} \mathbf{Q}_{X \times \Delta_{i}}(r) \longrightarrow \underset{i<j}{\oplus} \mathbf{Q}_{X \times \Delta_{i j}}(r) \longrightarrow \cdots \longrightarrow \underset{|I|=m}{\oplus} \mathbf{Q}_{X \times \Delta_{I}}(r),
$$

(2.2.6) and 2.2.7) follow from

$$
\operatorname{Ext}_{\operatorname{MHM}(Y)}^{\nu}\left(i_{Z *} \mathbf{Q}_{Z}(0), i_{I} * \mathbf{Q}_{X \times \Delta_{I}}(r)\right)=0 \quad \text { for } \forall I \text { and } \forall \nu<2 r
$$

where $i_{I}: X \times \Delta_{I} \hookrightarrow Y$, and

the composition of $c_{z}$ with $\mathbf{Q}_{Y}(r) \longrightarrow i_{*} \mathbf{Q}_{X \times \Delta_{i}}(r)$ is zero for $0 \leq \forall i \leq m$.

(2.2.9) follows from 2.2.5). In order to see (2.2.8), we have

$$
\begin{aligned}
\operatorname{Ext}_{\mathrm{MHM}(Y)}^{\nu}\left(i_{Z *} \mathbf{Q}_{Z}(0), i_{I *} \mathbf{Q}_{X \times \Delta_{I}}(r)\right) & =\operatorname{Ext}_{\mathrm{MHM}\left(X \times \Delta_{I}\right)}^{\nu}\left(i_{Z_{I} *} \mathbf{Q}_{Z_{I}}(0), \mathbf{Q}_{X \times \Delta_{I}}(r)\right) \\
& =\operatorname{Ext}_{\mathrm{MHM}\left(X \times \Delta_{I}\right)}^{\nu}\left(i_{Z_{I} *} \mathbf{Q}_{Z_{I}}(0), i_{Z_{I} *} i_{Z_{I}}^{!} \mathbf{Q}_{X \times \Delta_{I}}(r)\right)
\end{aligned}
$$

by the adjunction, where $Z_{I}:=Z \cap\left(X \times \Delta_{I}\right)$. Then (2.2.8) follows from the fact that $H^{k}\left(\left(i_{Z_{I} *} \mathbf{Q}_{Z_{I}}(0)\right)=0\right.$ for $k>d_{Z_{I}}$ and $H^{k}\left(i_{Z_{I} *} i_{Z_{I}} \mathbf{Q}_{X \times \Delta_{I}}(r)\right)=0$ for $k<d_{X \times \Delta_{I}}+r$, which can be checked on the underlying perverse sheaves (cf. SaM3 (7.15)). Thus we have shown Lemma 2.8.

Lemma 2.9. Let $p: Y \rightarrow X$ be the projection. Then $p_{*} j_{!} j^{*} \mathbf{Q}_{Y}(r)=\mathbf{Q}_{X}(r)[-m]$. 
In fact, since $j=1 \times j_{0}: X \times\left(\Delta^{m} \backslash \cup_{i} \Delta_{i}\right) \hookrightarrow Y=X \times \Delta^{m}$, we can assume that $X=$ Speck. Then the assertion follows from [aM3 (7.6).

By Lemma 2.8 and Lemma 2.9, we have the morphism

$$
\mathbf{Q}_{X}(0)=p_{*} \mathbf{Q}_{Y}(0) \rightarrow p_{*} i_{Z *} \mathbf{Q}_{Z}(0) \rightarrow p_{*} j ! j^{*} \mathbf{Q}_{Y}(r)[2 r]=\mathbf{Q}_{X}(r)[2 r-m],
$$

which gives the cycle map 2.2.3).

2.5. The Carlson isomorphisms. The category MHS is not semi simple, and the Yoneda extension groups in MHS have the well known explicit description, due to Carlson ( $\mathrm{Ca} \|$ ).

Here we review it. Since MHS carries the neutral Tannakian structure, we have:

$$
\operatorname{Ext}_{\mathrm{MHS}}^{\bullet}\left(H_{1}, H_{2}\right)=\operatorname{Ext}_{\mathrm{MHS}}^{\bullet}\left(\mathbf{Q}(0), H_{1}^{*} \otimes H_{2}\right) .
$$

Theorem 2.10 (Carlson). Let $H=\left(H_{\mathbf{Q}}, W_{\bullet}, F^{\bullet}\right)$ be a graded polarizable $\mathbf{Q}$-mixed Hodge structure. Then

(1) $\operatorname{Ext}_{\mathrm{MHS}}^{1}(\mathbf{Q}(0), H)=\operatorname{Ext}_{\mathrm{MHS}}^{1}\left(\mathbf{Q}(0), W_{0} H\right)=W_{-1} H_{\mathbf{C}} / W_{-1} H_{\mathbf{C}} \cap\left(F^{0} W_{0} H_{\mathbf{C}}+\right.$ $\left.W_{0} H_{\mathbf{Q}}\right)$ Here an element $\xi \in W_{-1} H_{\mathbf{C}}$ corresponds to the following extension of graded polarizable mixed Hodge structures:

$$
0 \longrightarrow W_{0} H \longrightarrow \widetilde{H} \longrightarrow \mathbf{Q}(0) \longrightarrow 0,
$$

where $\widetilde{H}=\left(\widetilde{H}_{\mathbf{Q}}, W_{\bullet}, F^{\bullet}\right)$ is the mixed Hodge structure with $\widetilde{H}_{\mathbf{Q}}=W_{0} H_{\mathbf{Q}} \oplus$ $\mathbf{Q}(0)$, and the weight filtration $W_{0} \widetilde{H}_{\mathbf{Q}}=\widetilde{H}_{\mathbf{Q}}, W_{\ell} \widetilde{H}_{\mathbf{Q}}=W_{\ell} H_{\mathbf{Q}}(\ell \leq-1)$, and the Hodge filtration $F^{p} \widetilde{H}_{\mathbf{C}}=F^{p} W_{0} H_{\mathbf{C}}(p \geq 1), F^{q} \widetilde{H}_{\mathbf{C}}=F^{q} W_{0} H_{\mathbf{C}}+F^{0} \widetilde{H}_{\mathbf{C}}$ $(q \leq-1)$ and

$$
F^{0} \widetilde{H}_{\mathbf{C}}=F^{0} W_{0} H_{\mathbf{C}}+\mathbf{C} \cdot(\xi \oplus 1) .
$$

(2) $\operatorname{Ext}_{\mathrm{MHS}}^{\nu}(\mathbf{Q}(0), H)=0$ for $\nu \geq 2$. (This is a formal consequence of the fact that the functor $\operatorname{Ext}^{1}(\mathbf{Q}(0),-)$ is right exact, which follows from the above explicit description.)

Remark 2.11. The author learned the above formula from Morihiko Saito, which is more complicated than the one by J. Carlson. Originally, J. Carlson calculated the Yoneda extension groups in the category of mixed Hodge structures which are not necessarily graded polarizable, and obtained a simple description ([Ca]),

$$
\operatorname{Ext}^{1}(\mathbf{Q}(0), H)=W_{0} H_{\mathbf{C}} / F^{0} W_{0} H_{\mathbf{C}}+W_{0} H_{\mathbf{Q}} .
$$

In particular, if $H=H^{2 r-1}\left(X^{a n}, \mathbf{Q}(r)\right)$ for a complex projective nonsingular variety $X$,

$$
\operatorname{Ext}_{\mathrm{MHS}}^{1}(\mathbf{Q}(0), H)=J^{r}(X)_{\mathbf{Q}},
$$

where $J^{r}(X)_{\mathbf{Q}}=H_{\mathbf{C}} / F^{0} H_{\mathbf{C}}+H_{\mathbf{Q}}$ is the $r$-th intermediate Jacobian of $X$. Using the mixed Hodge modules, we can define the Abel-Jacobi map

$$
\rho: \mathrm{CH}^{r}(X)_{\mathrm{hom}} \longrightarrow J^{r}(X)_{\mathbf{Q}},
$$

where $\mathrm{CH}^{r}(X)_{\text {hom }}$ denotes the $\mathbf{Q}$-tensored Chow group of $X$ generated by algebraic cycles of codimension $r$ which are homologically equivalent to 0 .

Let

$$
\mathrm{CH}^{r}(X) \longrightarrow \operatorname{Ext}_{\mathbf{M H M}(X)}^{2 r}\left(\mathbf{Q}_{X}(0), \mathbf{Q}_{X}(r)\right) .
$$


be the cycle map as in Corollary 2.7. By the Leray spectral sequence for the structure morphism $f: X \rightarrow$ SpecC (Corollary 2.6) and the vanishing of Ext MHS $_{\text {S }}$ of deree $\geq 2$, we get the following commutative diagram:

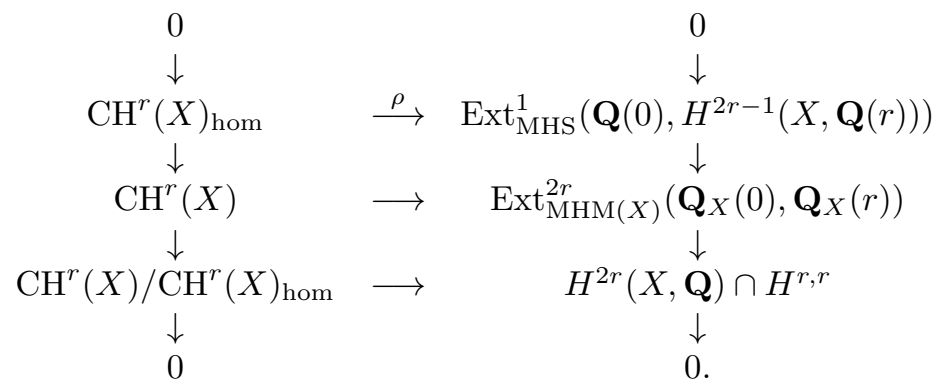

Then the top horizontal arrow $\rho$ is defined to be the Abel-Jacobi map.

The Abel-Jacobi maps were originally defined by A.Weil and P.Griffiths. Let us recall their definition.

Let $Z \in \mathrm{CH}^{r}(X)_{\text {hom }}$ be an algebraic cycle. There is a topological ( $2 \operatorname{dim} X-2 r+$ 1 )-cycle $\Gamma$ whose boundary is $Z: \partial \Gamma=Z$. Then the Abel-Jacobi class is defined as follows:

$$
\rho(Z)=\sum_{j=1}^{g}\left(\int_{\Gamma} \omega_{j}\right) \omega_{j}^{*} \in J^{r}(X)
$$

where $\omega_{1}, \cdots, \omega_{g} \in F^{n-r+1} H^{2 n-2 r+1}(X, \mathbf{C})$ is a basis, and $\omega_{1}^{*}, \cdots, \omega_{g}^{*} \in H^{2 r-1}(X, \mathbf{C}) / F^{r}$ denotes the Serre dual class of those: $\left\langle\omega_{i}, \omega_{j}^{*}\right\rangle=\delta_{i j}$.

It is known that the both definitions of Abel-Jacobi maps coincide (cf. [EZ], J3).

\section{Arithmetic Hodge structure}

Let $X$ be a nonsingular algebraic variety over $\mathbf{C}$. When we study the algebraic cycles of $X$ (in particular, contained in the kernel of the Abel-Jacobi map), the many difficulties are often caused from the fact of the vanishing of extension groups of mixed Hodge structure of degree $\geq 2$.

In this section, we introduce the notion of arithmetic Hodge structure (or more generally, arithmetic Hodge module), which has non-trivial higher extension groups.

3.1. Let $X$ be a quasi-projective nonsingular variety over $\mathbf{C}$. Then $X$ is defined by finitely many equations which possess finitely many coefficients. By considering the coefficients as parameters of a space $S$, we can obtain a model $f_{S}: X_{S} \rightarrow S$ and the Cartesian diagram:

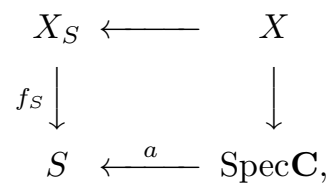

where $S$ is a nonsingular variety over $\overline{\mathbf{Q}}$, and the map $a$ factors through the generic point Spec $\overline{\mathbf{Q}}(S) \hookrightarrow S$. 
Definition 3.1. We define the abelian category

$$
\underline{\mathrm{M}}(X)=\underset{X_{S}}{\underset{\lim }{\longrightarrow}} \operatorname{HMM}\left(X_{S}\right) \text {. }
$$

Here $\operatorname{MHM}\left(X_{S}\right)$ is the category of mixed Hodge modules on the variety $X_{S}$ over $\overline{\mathbf{Q}}$. In the above limit, $X_{S}$ runs over all models (3.3.1), and for a morphism $j$ : $X_{S^{\prime}} \rightarrow X_{S}$ of the models, we take the pull-back $j^{*}: \operatorname{MHM}\left(X_{S}\right) \rightarrow \operatorname{MHM}\left(X_{S^{\prime}}\right)$. We call it the category of arithmetic Hodge modules. In particular, we call $\underline{\mathrm{M}}(\mathbf{C}):=$ $\underline{\mathrm{M}}(\mathrm{Spec} \mathbf{C})$ the category of arithmetic Hodge structures.

$\underline{\mathrm{M}}(X)$ is an abelian category. By Theorem 2.3 (画) and the remark about Definition 2.5, the category of arithmetic Hodge modules carries the standard functors:

$$
f_{*}, f_{!}, f^{*}, f^{!}, \mathbb{D}, \otimes, \underline{\text { Hom. }} .
$$

The arithmetic Tate Hodge module $\mathbf{Q}_{X}(r)$ is defined as the equivalence class of $\left[\mathbf{Q}_{X_{S}}(r)\right]$ of the Tate Hodge module on a model $X_{S}$. It does not depend on the choice of a model $X_{S}$. It follows from the fact that for any two models $X_{S}$ and $X_{S^{\prime}}$, there is another model $X_{S^{\prime \prime}}$ with morphisms $X_{S^{\prime \prime}} \rightarrow X_{S}$ and $X_{S^{\prime \prime}} \rightarrow X_{S^{\prime}}$ of models. We write $H^{\bullet}(X, \mathbf{Q}(r)):=R^{\bullet} f_{*} \mathbf{Q}_{X}(r)$ (or, simply $H^{\bullet}(X)(r)$ ) for the structure morphism $f: X \rightarrow$ SpecC.

3.2. The space of Mumford's infinitesimal invariants. Yoneda extension groups in the category of arithmetic Hodge structures are complicated. I do not know its explicit form in general. Here we construct a natural map from the extension groups to the spaces of Mumford's infinitesimal invariants, which have explicit forms.

Let $X$ be a nonsingular projective variety over $\mathbf{C}$. Let us recall the arithmetic Gauss-Manin connection on the algebraic de Rham cohomology (cf. $[\mathrm{H}-\mathrm{dR}$ ):

$$
\nabla: H_{d R}^{q}(X / \mathbf{C}) \longrightarrow H_{d R}^{q}(X / \mathbf{C}) \otimes_{\mathbf{C}} \Omega_{\mathbf{C} / \overline{\mathbf{Q}}}^{1}
$$

Let $f: X \rightarrow$ Spec $\mathbf{C}$ be the structure morphism. The exact sequence

$$
0 \longrightarrow f^{*} \Omega_{\mathbf{C} / \overline{\mathbf{Q}}}^{1} \longrightarrow \Omega_{X / \overline{\mathbf{Q}}}^{1} \longrightarrow \Omega_{X / \mathbf{C}}^{1} \longrightarrow 0
$$

induces the following exact sequence

$$
0 \longrightarrow \Omega_{X / \mathbf{C}}^{\bullet-1} \otimes f^{*} \Omega_{\mathbf{C} / \overline{\mathbf{Q}}}^{1} \longrightarrow \Omega_{X / \overline{\mathbf{Q}}}^{\bullet} / U^{2} \longrightarrow \Omega_{X / \mathbf{C}}^{\bullet} \longrightarrow 0,
$$

where $U^{2}:=\operatorname{Image}\left(\Omega_{X / \overline{\mathbf{Q}}}^{\bullet-2} \otimes f^{*} \Omega_{\mathbf{C} / \overline{\mathbf{Q}}}^{2} \rightarrow \Omega_{X / \overline{\mathbf{Q}}}^{\bullet}\right)$. Applying $R f_{*}$ on (3.3.3), we obtain the coboundary map $H_{d R}^{q}(X / \mathbf{C}):=R^{q} f_{*} \Omega_{X / \mathbf{C}}^{\bullet} \rightarrow R^{q} f_{*}\left(\Omega_{X / \mathbf{C}}^{\bullet-1} \otimes f^{*} \Omega_{\mathbf{C} / \overline{\mathbf{Q}}}\right) \simeq$ $H_{d R}^{q}(X / \mathbf{C}) \otimes \Omega_{\mathbf{C} / \overline{\mathbf{Q}}}^{1}$ (where the last isomorphism is the projection formula), and hence the arithmetic Gauss-Manin connection (3.3.2).

By definition, the arithmetic Gauss-Manin connection (3.3.2) satisfies the Griffiths transversality

$$
\nabla\left(F^{p}\right) \subset F^{p-1} \otimes \Omega_{\mathbf{C} / \overline{\mathbf{Q}}}^{1},
$$

where $F^{s}=F^{s} H_{d R}^{q}(X / \mathbf{C})=H^{q}\left(X, \Omega_{X / \mathbf{C}}^{\bullet \geq s}\right)$ denotes the Hodge filtration.

Definition 3.2. Let $p, q, r$ be integers. Then $\Xi_{X}^{p, q}(r)$ is defined as the cohomology at the middle term of the following complex induced from the arithmetic GaussManin connection (3.3.2):

$F^{p+1} H_{d R}^{p+q}(X / \mathbf{C}) \otimes \Omega_{\mathbf{C} / \overline{\mathbf{Q}}}^{r-1} \stackrel{\nabla}{\rightarrow} F^{p} H_{d R}^{p+q}(X / \mathbf{C}) \otimes \Omega_{\mathbf{C} / \overline{\mathbf{Q}}}^{r} \stackrel{\nabla}{\rightarrow} F^{p-1} H_{d R}^{p+q}(X / \mathbf{C}) \otimes \Omega_{\mathbf{C} / \overline{\mathbf{Q}}}^{r+1}$. 
We construct a natural map from the extension groups in $\underline{\mathrm{M}}(\mathbf{C})$ to the space $\Xi_{X}^{p, q}(r)$

Let $X_{S} \rightarrow S$ be the model of $X$ (3.3.1). Recall the category $\operatorname{MHM}(S)$ is defined to be a full subcategory of the fiber product $\operatorname{MFW}_{r h}(S) \times_{\mathrm{MFW}_{r h}\left(S_{\mathbf{C}}\right)} \operatorname{MHM}\left(S_{\mathbf{C}}\right)$ such that the polarization on each weight quotient is defined over $\mathbf{Q}$. There is the forgetful functor $\operatorname{MHM}(S) \rightarrow \mathrm{MF}_{r h}(S)$, which is exact by Theorem 2.3 (2):

$$
\operatorname{MHM}(S) \longrightarrow \operatorname{MF}_{r h}(S), \quad\left(K_{\mathbf{Q}}^{\bullet}, M, F, W\right) \mapsto(M, F) .
$$

Therefore we have the well-defined map of Yoneda extension groups:

$$
\operatorname{Ext}_{\mathrm{MHM}(S)}^{p}\left(\mathbf{Q}_{S}(0), R^{q} f_{*} \mathbf{Q}_{X_{S}}(r)\right) \longrightarrow \operatorname{Ext}_{\mathrm{MF}_{r h}(S)}^{p}\left(\mathcal{O}_{S}, H_{d R}^{q}\left(X_{S} / S\right)(r)\right)
$$

Note that $\operatorname{MF}_{r h}(S)$ is an exact category (see. $\S 2.2$ ), and we can define its derived category $D^{b}\left(\mathrm{MF}_{r h}(S)\right)$ (cf. BBD 1.1). Then we can describe $\operatorname{Ext}_{\mathrm{MF}_{r h}(S)}^{p}(A, B)=$ $\operatorname{Hom}_{D^{b}\left(\operatorname{MF}_{r h}(S)\right)}(A, B[p])$. We will take the inductive limit over $S$ of the right hand side in (3.3.5). So we may assume $S=\operatorname{Spec} \mathcal{O}$ where $\mathcal{O}$ is a regular local ring over $\overline{\mathbf{Q}}$ with regular parameter $x_{1}, \cdots, x_{n}$. Then we can see $D_{S}=\mathcal{O}\left[\partial_{1}, \cdots, \partial_{n}\right]$ where $\partial_{i}$ is the derivation on $\mathcal{O}$ over $\overline{\mathbf{Q}}$ such that $\left[\partial_{i}, x_{j}\right]=\delta_{i j}$. Let $D_{S}(m)$ be the filtered $D$-module $\left(D_{S}, F_{\bullet+m}\right)$ with the differential order filtration shifted by $m$.

Lemma 3.3. $\operatorname{Ext}_{\mathrm{MF}_{r h}(S)}^{p}\left(D_{S}(m), M\right)=0 \quad \forall p \geq 1$.

Proof. Standard.

There is the Koszul resolution of $\mathcal{O}_{S}$

$$
0 \rightarrow D_{S}(-n) \otimes_{\overline{\mathbf{Q}}} \stackrel{n}{\wedge} V \rightarrow \cdots \rightarrow D_{S}(-1) \otimes_{\overline{\mathbf{Q}}} V \rightarrow D_{S} \rightarrow \mathcal{O}_{S} \rightarrow 0,
$$

where $V$ is a $n$-dimensional $\overline{\mathbf{Q}}$-vector space with a basis $e_{1}, \cdots, e_{n}$, and the boundary map $D_{S}(-p) \otimes_{\overline{\mathbf{Q}}} \stackrel{p}{\wedge} V \rightarrow D_{S}(-p+1) \otimes_{\overline{\mathbf{Q}}} \stackrel{p-1}{\wedge} V$ is given by $\xi \otimes e_{i_{1}} \wedge \cdots \wedge e_{i_{p}} \mapsto$ $\sum_{j=1}^{p}(-1)^{j} \xi \cdot \partial_{i_{j}} \otimes e_{i_{1}} \wedge \cdots \wedge \hat{e}_{i_{j}} \wedge \cdots \wedge e_{i_{p}}$. It is also the exact sequence in the exact category $\operatorname{MF}_{r h}(S)$.

Using the exact sequence (3.3.6) and applying Lemma 3.3, we can see that the right hand side of (3.3.5) is isomorphic to the cohomology at the middle term of the following complex induced from the algebraic Gauss-Manin connection:

$F^{r-p+1} H_{d R}^{q}\left(X_{S} / S\right) \otimes \Omega_{S / \overline{\mathbf{Q}}}^{p-1} \stackrel{\nabla}{\rightarrow} F^{r-p} H_{d R}^{q}\left(X_{S} / S\right) \otimes \Omega_{S / \overline{\mathbf{Q}}}^{p} \stackrel{\nabla}{\rightarrow} F^{r-p-1} H_{d R}^{q}\left(X_{S} / S\right) \otimes \Omega_{S / \overline{\mathbf{Q}}}^{p+1}$.

Passing to the limit over $S$, we thus have:

Proposition 3.4. Let $X$ be a nonsingular projective variety over $\mathbf{C}$. Then there is the following natural map:

$$
\operatorname{Ext}_{\underline{\mathbf{M}}(\mathbf{C})}^{p}\left(\mathbf{Q}(0), H^{q}(X)(r)\right) \longrightarrow \Xi_{X}^{r-p, q-r+p}(p) .
$$

Definition 3.5. Let $p, q, r$ be integers. The space of Mumford's infinitesimal invariants $\Lambda_{X}^{p, q}(r)$ is defined as the cohomology at the middle term of the following complex induced from the arithmetic Gauss-Manin connection (3.3.2):

$$
H^{q-1}\left(\Omega_{X / \mathbf{C}}^{p+1}\right) \otimes \Omega_{\mathbf{C} / \overline{\mathbf{Q}}}^{r-1} \stackrel{\bar{\nabla}}{\rightarrow} H^{q}\left(\Omega_{X / \mathbf{C}}^{p}\right) \otimes \Omega_{\mathbf{C} / \overline{\mathbf{Q}}}^{r} \stackrel{\bar{\nabla}}{\rightarrow} H^{q+1}\left(\Omega_{X / \mathbf{C}}^{p-1}\right) \otimes \Omega_{\mathbf{C} / \overline{\mathbf{Q}}}^{r+1} .
$$


Clearly, there is the natural map,

$$
\Xi_{X}^{p, q}(r) \longrightarrow \Lambda_{X}^{p, q}(r)
$$

By the similar argument to the above, we can see

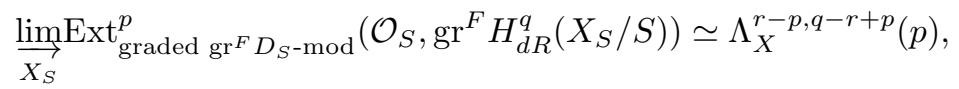

and the map (3.3.7) is compatible to the map between Yoneda extension groups induced from the exact functor $\mathrm{MF}_{r h}(S) \rightarrow$ \{graded $\operatorname{gr}^{F} D_{S}$-mod $\},(M, F) \mapsto \operatorname{gr}^{F} M$.

Together with Proposition 3.4, we thus have:

Proposition 3.6. Let $X$ be a nonsingular projective variety over $\mathbf{C}$. Then we have the natural map from the extension groups in $\underline{\mathrm{M}}(\mathbf{C})$ to the space of Mumford's infinitesimal invariants as the composition of the map in Proposition 3.4 and (3.3.7):

$$
\operatorname{Ext}_{\underline{\mathbf{M}}(\mathbf{C})}^{p}\left(\mathbf{Q}(0), H^{q}(X)(r)\right) \longrightarrow \Lambda_{X}^{r-p, q-r+p}(p) .
$$

\section{Algebraic cycles and extensions of arithmetic Hodge structure}

In this section, we study the extension groups in the abelian category $\underline{\mathrm{M}}(\mathbf{C})$ and algebraic cycles, in particular, the Bloch conjecture.

4.1. Higher Abel-Jacobi maps. Let $X$ be a nonsingular variety over C. We denote its higher Chow group by $\mathrm{CH}^{r}(X, m)$. Then we have the following map

$$
\begin{gathered}
\underset{X_{S}}{\lim _{\longrightarrow}} \mathrm{CH}^{r}\left(X_{S}, m\right) \longrightarrow \underset{X_{S}}{\lim } \operatorname{Ext}_{\operatorname{MHM}\left(X_{S}\right)}^{2 r-m}\left(\mathbf{Q}_{X_{S}}(0), \mathbf{Q}_{X_{S}}(r)\right) \\
\| \\
\mathrm{CH}^{r}(X, m) \longrightarrow \operatorname{Ext}_{\underline{\underline{M}}(X)}^{2 r-m}\left(\mathbf{Q}_{X}(0), \mathbf{Q}_{X}(r)\right),
\end{gathered}
$$

where the top horizontal arrow is the one in Corollary 2.7. We denote the above map by $c=c^{r}(X, m)$. On the other hand, there is the Leray spectral sequence

$$
E_{2}^{p q}=\operatorname{Ext}_{\underline{\mathbf{M}}(\mathbf{C})}^{p}\left(\mathbf{Q}(0), H^{q}(X)(r)\right) \Longrightarrow \operatorname{Ext}_{\underline{\mathbf{M}}(X)}^{p+q}\left(\mathbf{Q}_{X}(0), \mathbf{Q}_{X}(r)\right),
$$

as the inductive limit of the Leray spectral sequence for the model $X_{S}$ (Corollary 2.6). It degenerates at $E_{2}$ terms if $X$ is projective. The spectral sequence (4.4.2) defines the Leray filtration on the extension group $\operatorname{Ext}_{\underline{\underline{M}}(X)}\left(\mathbf{Q}_{X}(0), \mathbf{Q}_{X}(r)\right)$, which we denote by $F^{\bullet}$.

Definition 4.1. Let $X$ be a nonsingular projective variety over $\mathbf{C}$. We define the filtration on the higher Chow group as follows:

$$
F^{\nu} \mathrm{CH}^{r}(X, m):=c^{-1} F^{\nu} \operatorname{Ext}_{\underline{\mathbf{M}}(X)}^{2 r-m}\left(\mathbf{Q}_{X}(0), \mathbf{Q}_{X}(r)\right) .
$$

Then, together with the $E_{2}$-degeneration of the spectral sequence 4.4.2), we obtain:

$$
\rho_{X}^{\nu}: \operatorname{Gr}_{F}^{\nu} \mathrm{CH}^{r}(X, m) \longrightarrow \operatorname{Ext}_{\underline{M}(\mathbf{C})}^{\nu}\left(\mathbf{Q}(0), H^{2 r-m-\nu}(X)(r)\right) .
$$

We call the above the $\nu$-th higher Abel-Jacobi map.

By definition, we can easily show Theorem 1.1(11) and (2). For example, Theorem 1.1 (2) (d) can be shown by using the argument in [Mur2. Theorem 1.1 (2) (e) follows from the hard Lefschetz theorem in the terminology of arithmetic Hodge structure. (See [A] for details.) 
Example 4.2. Let $r=\operatorname{dim} X=n, \nu=2$ and $m=0$. Then the higher Abel-Jacobi map gives the following map:

$$
\rho_{X}^{2}: T(X) \longrightarrow \operatorname{Ext}_{\underline{\mathrm{M}}(\mathbf{C})}^{2}\left(\mathbf{Q}(0), H^{2 n-2}(X)(n)\right) .
$$

We call the above (4.4.3) the second Albanese map.

4.2. Mumford's infinitesimal invariants. Composing the higher Abel-Jacobi map and the maps in Proposition 3.4 and 3.6, we have Theorem 1.1 (35):

$$
\begin{aligned}
& \xi_{X}^{\nu}: \operatorname{Gr}_{F}^{\nu} \mathrm{CH}^{r}(X, m) \longrightarrow \Xi_{X}^{r-\nu, r-m}(\nu), \\
& \delta_{X}^{\nu}: \operatorname{Gr}_{F}^{\nu} \mathrm{CH}^{r}(X, m) \longrightarrow \Lambda_{X}^{r-\nu, r-m}(\nu) .
\end{aligned}
$$

When $m=0$, the above maps have already been defined in [AS].

We know the map of infinitesimal invariants $\delta_{X}$ is not zero in general. More strongly, the following holds.

Theorem 4.3 ([AS] Theorem (0-6)). Let $X \subset \mathbf{P}_{\mathrm{C}}^{m+s}$ be a nonsingular complete intersection variety of degree $\left(d_{1}, \cdots, d_{s}\right)$, with a sufficiently general coefficients. Assume that one of the following conditions holds.

(1) $m \geq 2$ and $\min \left\{d_{i}\right\} \cdot s \geq 2 m+s$.

(2) $m=1$ and $\min \left\{d_{i}\right\} \cdot s \geq 3+s$.

Then the map of infinitesimal invariant induces an injective map

$$
\delta_{X}^{m}: F^{m} \mathrm{CH}^{m}(X)_{\text {gen }} \longrightarrow \Lambda_{X}^{0, m}(m) .
$$

Here $F^{m} \mathrm{CH}^{m}(X)_{\text {gen }}$ denotes the subgroup of $F^{m} \mathrm{CH}^{m}(X)$ generated by 0 -cycles in general positions (loc.cit. Ch.II Definition (5-4-1)). In particular, we have

$$
F^{m+1} \mathrm{CH}^{m}(X)_{\text {gen }}=0 \text {. }
$$

Remark 4.4. For a nonsingular complete intersection $X$ of dimension $m$, it is easy to see that $F^{2} \mathrm{CH}^{m}(X)=\cdots=F^{m} \mathrm{CH}^{m}(X)=T(X)$ and $\delta_{X}^{m}\left(F^{m+1} \mathrm{CH}^{m}(X)\right)=0$, where $F^{\bullet}$ denotes either our filtration or S.Saito's filtration (AS] Ch.II $\S 2$, SaS1], SaS2 2). It is conjectured that our filtration coincides with S.Saito's filtration. But I do not have the proof of it.

Proof. (Sketch). We prove the assertion by the induction of $\operatorname{dim} X$.

We first show the map

$$
\delta_{C}^{1}: F^{1} \mathrm{CH}^{1}(C) \longrightarrow \Lambda_{C}^{0,1}(1) .
$$

is injective for a general complete intersection curve $C \subset \mathbf{P}_{\mathbf{C}}^{s+1}$ of degree $\left(d_{1}, \cdots, d_{s}\right)$, which satisfies $\min \left\{d_{i}\right\} \cdot s \geq 3+s$. Let $f: C_{S} \rightarrow S$ be a model of $C$, where we may assume that there is a dominant morphism $S \rightarrow \mathcal{M}$ to the moduli space of complete intersection curves of degree $\left(d_{1}, \cdots, d_{s}\right)$, because the coefficients of $C$ is general. Let $\Lambda_{C_{S} / S}^{0,1}(1):=\Gamma\left(S, R^{1} f_{*} \mathcal{O}_{C_{S}} \otimes \Omega_{S / \overline{\mathbf{Q}}}^{1} / \bar{\nabla}\left(f_{*} \Omega_{C_{S} / S}^{1}\right)\right)$. Then the map (4.4.5) can be obtained by the inductive limit over $S$ of the map $F_{S}^{1} \mathrm{CH}^{1}\left(C_{S}\right) \rightarrow \Lambda_{C_{S} S}^{0,1}(1)$. Here $F_{S}^{\bullet} \mathrm{CH}^{\bullet}\left(C_{S}\right)$ denotes the relative filtration on the Chow group (cf. AS] Ch.II $\S 2$ ). Let $\Lambda_{C_{S}^{a n} / S^{a n}}^{0,1}(1):=\Lambda_{C_{S} / S}^{0,1}(1) \otimes \mathcal{O}_{S}^{a n} \simeq \Gamma\left(S^{a n}, R^{1} f_{*} \mathcal{O}_{C_{S}} \otimes \Omega_{S^{a n} / \mathbf{C}}^{1} / \bar{\nabla}\left(f_{*} \Omega_{C_{S}^{a n} / S^{a n}}^{1}\right)\right)$. 
In order to prove the injectivity of (4.4.5), it suffices to show that if a cycle $z \in F_{S}^{1} \mathrm{CH}^{1}\left(C_{S}\right)$ has a zero-image under the following map

$$
\delta_{S}^{1}: F_{S}^{1} \mathrm{CH}^{1}\left(C_{S}\right) \longrightarrow \Lambda_{C_{S}^{a n} / S^{a n}}^{0,1}(1),
$$

then $\left.z\right|_{C}$ is rationally equivalent to zero. Since $f_{*} \Omega_{C_{S}^{a n} / S}^{1} \otimes \Omega_{S^{a n} / \mathbf{C}}^{1} \rightarrow R^{1} f_{*} \mathcal{O}_{C_{S}} \otimes$ $\Omega_{S^{a n} / \mathrm{C}}^{2}$ is injective by the symmetrizer lemma for complete intersections (loc.cit.Ch.I.Corollary $(4-5))$, we have the injection

$$
\begin{aligned}
\Xi_{C_{S}^{a n} / S^{a n}}^{0,1}(1) & :=\Gamma\left(S^{a n},\left(R^{1} f_{*} \Omega_{C_{S}^{a n} / S^{a n}}^{\bullet}\right) \otimes \Omega_{S^{a n} / \mathbf{C}}^{1} / \nabla\left(f_{*} \Omega_{C_{S}^{a n} / S^{a n}}^{1}\right)\right) \\
& \hookrightarrow \Lambda_{C_{S}^{a n} / S^{a n}}^{0,1}(1) .
\end{aligned}
$$

There is the following exact sequence

$$
0 \rightarrow \Gamma\left(S^{a n}, R^{1} f_{*} \mathbf{C}_{C_{S}^{a n}} / R^{1} f_{*} \mathbf{Q}_{C_{S}^{a n}}\right) \rightarrow \Gamma\left(S^{a n}, J\left(C_{S} / S\right)\right) \rightarrow \Xi_{C_{S}^{a n} / S^{a n}}^{0,1}(1),
$$

where we put $J\left(C_{S} / S\right):=R^{1} f_{*} \Omega_{C_{S}^{a n} / S^{a n}}^{\bullet} / f_{*} \Omega_{C_{S}^{a n} / S^{a n}}^{1}+R^{1} f_{*} \mathbf{Q}_{C_{S}^{a n}}$. It is easy to see that $\Gamma\left(S^{a n}, R^{1} f_{*} \mathbf{C}_{C_{S}^{a n}}\right)=0$ and hence $\Gamma\left(S^{a n}, R^{1} f_{*} \mathbf{C}_{C_{S}^{a n}} / R^{1} f_{*} \mathbf{Q}_{C_{S}^{a n}}\right)=0$ because of the dominant morphism $S \rightarrow \mathcal{M}$. Thus it follows from the Abel's theorem that if $\delta_{S}(z)=0$, then $\left.z\right|_{f^{-1}(t)}=0$ for any $t \in S(\mathbf{C})$, in particular, $\left.z\right|_{C}=0$.

Next we prove for any dimensional case. Let $X$ be as in Theorem 4.3 and $m \geq 2$. let

$$
X=X_{m} \supset X_{m-1} \supset \cdots \supset X_{2} \supset X_{1}=C
$$

be a sequence of general hyperplane sections, in which the multi-degree of each $X_{i}$ satisfies the condition in Theorem 4.3. Then there is the commutative diagram

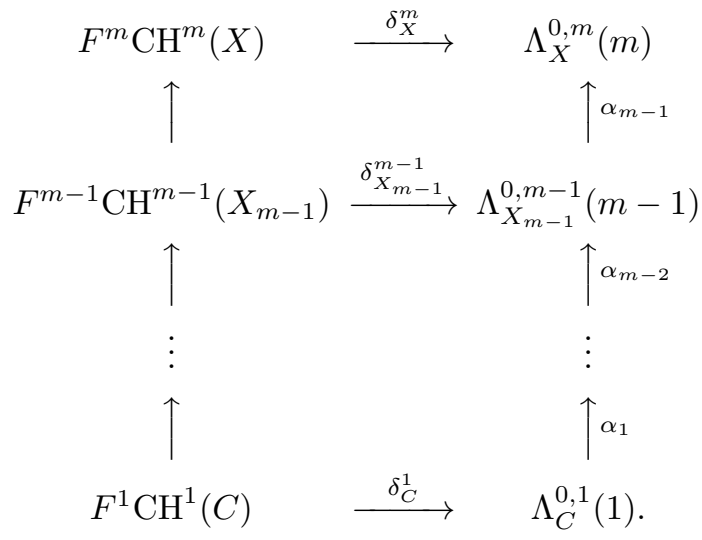

By the above, $\delta_{C}^{1}$ is injective. Moreover, each $\alpha_{i}$ is also injective by the symmetrizer lemma for open complements (loc.cit. Ch.I Theorem (4-4)). Put

$$
F^{m} \mathrm{CH}^{m}(X)_{\text {gen }}:=\operatorname{Image}\left(\sum_{C} F^{1} \mathrm{CH}^{1}(C) \rightarrow F^{m} \mathrm{CH}^{m}(X)\right)
$$

where $C$ runs over all curves as above. Then we have that the map 4.4.4)

$$
\delta_{X}^{m}: F^{m} \mathrm{CH}^{m}(X)_{\text {gen }} \longrightarrow \Lambda_{X}^{0, m}(m)
$$

is injective. 
Mumford's infinitesimal invariants have the advantage of explicit calculations. But it is a "coarse" invariant, that is, there are several examples of an algebraic cycle which is not rationally equivalent to zero, but its infinitesimal invariant is zero (cf. G2 ). We hope that our higher Abel-Jacobi invariants capture all cycles (cf. Conjecture 4.7 below). In fact, there is an example of a 0-cycle on a surface such that its infinitesimal invariant vanishes, but the higher Abel-Jacobi invariant does not:

Theorem 4.5. Let $C$ be a projective nonsingular curve over $\overline{\mathbf{Q}}$ with genus $g \geq 2$, such that $\operatorname{rankNS}(C \times C)=3$ 开. Let $O \in C(\overline{\mathbf{Q}})$ be a $\overline{\mathbf{Q}}$-valued point such that $K_{C}-(2 g-2) \cdot O$ is not $\mathbf{Q}$-linearly equivalent to 0 5. Let $P \in C(\mathbf{C}) \backslash C(\overline{\mathbf{Q}})$ be any $\mathbf{C}$-valued point which is not $\overline{\mathbf{Q}}$-valued one. Put $X:=C_{\mathbf{C}} \times C_{\mathbf{C}}$ and $z:=$ $(P, P)-(P, O)-(O, P)+(O, O) \in T(X)$. Then we have

$$
\xi_{X}^{2}(z)=0 \quad \text { in } \quad \Xi_{X}^{0,2}(2),
$$

but

$$
\rho_{X}^{2}(z) \neq 0 \quad \text { in } \operatorname{Ext}_{\underline{\mathbf{M}}(\mathbf{C})}^{2}\left(\mathbf{Q}(0), H^{2}(X)(2)\right) .
$$

The proof of the above theorem will appear in the forthcoming paper [A].

4.3. Bloch conjecture. Recall the famous Bloch conjecture (B1] Lec.1):

Conjecture 4.6 (Bloch). Let $X$ be a projective nonsingular surface over $\mathbf{C}$ with the geometric genus $p_{g}=\operatorname{dim} H^{0}\left(K_{X / \mathbf{C}}\right)=0$. Then the (usual) Albanese map

$$
A_{0}(X) \longrightarrow \operatorname{Alb}(X)
$$

is injective.

This conjecture was observed by S.Bloch with an ingenious insight, and it led to the Bloch-Beilinson formula (1.1.1). The Bloch conjecture was proved for surfaces which are not of general type (BKL). But, in general, it is still open.

Now we conjecture:

Conjecture 4.7. The second Albanese map (4.4.3)

$$
\rho_{X}^{2}: T(X) \longrightarrow \operatorname{Ext}_{\underline{\underline{M}}(\mathbf{C})}^{2}\left(\mathbf{Q}(0), H^{2}(X)(2)\right)
$$

is injective for any projective nonsingular surface $X$ (not necessarily $p_{g}=0$ ).

Or, more generally

Conjecture 4.8. The cycle map $c=c^{r}(X, m)$ in (4.4.1) is injective for any projective nonsingular variety $X$ over $\mathbf{C}$.

Conjecture 4.7 implies the Bloch conjecture (use the method in [B1] Lec. 1). Moreover Conjecture 4.8 also has an application to algebraic cycles on varieties over an algebraic number field. Let us explain it.

Let $X_{\overline{\mathbf{Q}}}$ be a nonsingular projective variety over $\overline{\mathbf{Q}}$. By the construction, the higher Abel-Jacobi map factors as follows:

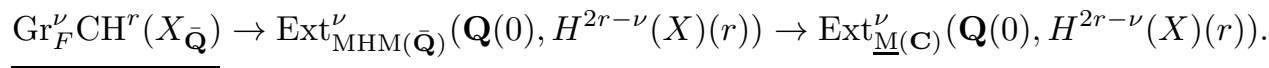

4 The proof of the existence of such a curve was taught from S.Mochizuki (the private communication with the author).

5 There are infinitely many such points $O$ by Raynaud's theorem ( $\mathrm{B}]$ ). 
Here $F^{\nu} \mathrm{CH}^{r}\left(X_{\overline{\mathbf{Q}}}\right)$ denotes the subgroup of $F^{\nu} \mathrm{CH}^{r}\left(X_{\mathbf{C}}\right)$ generated by cycles of codimension $r$ defined over $\overline{\mathbf{Q}}$. Since the arithmetic Gauss-Manin connection in $\operatorname{MHM}(\overline{\mathbf{Q}})$ vanishes, we can show any extension groups in $\operatorname{MHM}(\overline{\mathbf{Q}})$ vanishes if $\nu \geq 2$ (cf. Theorem 2.10 (2)). Then, due to Conjecture 4.8, we have

$$
F^{2} \mathrm{CH}^{r}\left(X_{\overline{\mathbf{Q}}}\right)=0 \text {. }
$$

In particular,

$$
T\left(X_{\overline{\mathbf{Q}}}\right)=0 .
$$

Let $X_{k}$ be a nonsingular projective variety over an algebraic number field $k$. Due to the injectivity of the map $\mathrm{CH}_{0}\left(X_{k}\right) \rightarrow \mathrm{CH}_{0}\left(X_{\overline{\mathbf{Q}}}\right)$ (note that the Chow groups are always tensored with $\mathbf{Q})$, we also have $T\left(X_{k}\right)=0$. Combined with the MordellWeil theorem, we obtain the finiteness of the rank of the Chow group:

$$
\operatorname{rank} \mathrm{CH}_{0}\left(X_{k}\right)<\infty \text {. }
$$

Theorem 4.9. (1) Conjecture 4.7 implies the Bloch conjecture 4.6.

(2) Let $X_{k}$ be a nonsingular projective variety over an algebraic number field $k$. Assume that Conjecture 4.8 holds. Then we have $F^{2} \mathrm{CH}^{r}\left(X_{\overline{\mathbf{Q}}}\right)=0$. In particular, it implies that the rank of $\mathrm{CH}_{0}\left(X_{k}\right)$ is finite.

Further we can show the 'almost' converse of Theorem 4.9 (2) for $r=2$ :

Theorem 4.10 (这). Assume the followings hold:

(1) The Abel-Jacobi map (cf. §2.5)

$$
\rho: \mathrm{CH}^{2}\left(X_{\overline{\mathbf{Q}}}\right)_{\text {hom }} \longrightarrow J^{2}\left(X_{\mathbf{C}}\right)
$$

is injective for any projective nonsingular variety $X_{\overline{\mathbf{Q}}}$ of arbitrary dimension over $\overline{\mathbf{Q}}$. (Here we put $X_{\mathbf{C}}=X_{\overline{\mathbf{Q}}} \otimes \mathbf{C}$.)

(2) Let $X_{\overline{\mathbf{Q}}}$ and $\rho$ be as above. Let $z \in \mathrm{CH}^{2}\left(X_{\mathbf{C}}\right)_{\text {hom }}$ be any algebraic cycle. Then $\rho(z)=0$ if and only if $\rho\left(z^{\sigma}\right)=0$ for any $\sigma \in \operatorname{Aut}(\mathbf{C} / \overline{\mathbf{Q}})$. (Note that $\operatorname{Aut}(\mathbf{C} / \overline{\mathbf{Q}})$ acts on $\mathrm{CH}^{2}\left(X_{\mathbf{C}}\right)_{\text {hom }}$.)

Then Conjecture 4.8 for $r=2$ and $m=0$ is true. In particular, (1) and (2) implies the Bloch conjecture 1.6 .

Theorem 4.10 (1) is nothing but the conjecture due to A.Beilinson. Theorem 4.10 (2) should be true if $F_{\mathcal{M}}^{2}=\operatorname{ker} \rho$.

4.4. Finally we propose the following problem about surjectivity of the cycle map.

Let $X$ be a projective nonsingular variety over $\mathbf{C}$, and put

$$
J^{r}(X)_{\text {arith }}=\operatorname{Image}\left(\operatorname{Ext}_{\underline{\underline{M}}(\mathbf{C})}^{1}\left(\mathbf{Q}(0), H^{2 r-1}(X)(r)\right) \longrightarrow J^{r}(X)_{\mathbf{Q}}\right)
$$

for $r \geq 2$ (cf. 2.5 ).

Problem 4.11. Is the Abel-Jacobi map

$$
\mathrm{CH}^{r}(X)_{\mathrm{hom}} \longrightarrow J^{r}(X)_{\text {arith }}
$$

surjective?

In general, the Abel-Jacobi map (2.2.11) is not surjective. For example, when the Hodge number $\Sigma_{k \geq r+1} h^{k, 2 r-1-k}(X) \neq 0$, it is not so (or, more strong result, see [G1]). It is an interesting problem to give Hodge theoretic characterization of its image. 


\section{REFERENCES}

[BBD] A.Beilinson, J.Bernstein and P.Deligne, Faisceaux Pervers. Astérisque 100 (1983).

[B1] S.Bloch, Lectures on algebraic cycles, Duke Univ. Math. Ser. Vol IV, Duke Univ. Durham, NC, 1980.

[B2] - Algebraic cycles and higher K-theory, Adv. in Math. 61 (1986), 267-304.

[BKL] Bloch, Kas and Lieberman, Zero cycles on surfaces with $p_{g}=0$, Compositio. Math. 33 (1976), 135-145.

[Ca] J.Carlson, Extensions of mixed Hodge structures, Journées de geométrie algébrique d'Angers 1979, Sijthoff and Noordhoff, pp.107-127.

[D1] P.Deligne, Théorie de Hodge. II, Publ. Math. IHES, 40 (1971), 5-58.

[D2] - Théorie de Hodge. III, Publ. Math. IHES, 44 (1975), 5-77.

[D3] Equations différentielles à points singuliers réguliers, Lecture note in Math. 163, Springer, 1970.

[DMOS] P.Deligne et al, Hodge cycles, Motives and Shimura Varieties, Lecture note in Math. 900, Springer, 1982.

[EZ] F.El Zein and S.Zucker, Extendability of normal functions associated to algebraic cycles, in Topics in transcendental algebraic geometry, edited by P.Griffiths, Princeton, 1984 pp.269-288.

[G1] M.Green, Griffiths infinitesimal invariant and the Abel-Jacobi map, J. Diff. Geom. 29 (1989), 545-555.

[G2] Algebraic cycles and Hodge theory, to appear in this proceedings.

[H-dR] R.Hartshorne, On the de Rham cohomology of algebraic varieties, Publ. Math. IHES, 45 (1976), 5-99.

[J1] U.Jannsen, Mixed Motives and Algebraic K-theory, Lecture note in Math. 1400, Springer, 1990.

[J2] Motivic sheaves and filtration on Chow groups, In Motives I, Proc. Sympos. Pure Math. vol.55, AMS, 1994, pp.245-302.

[J3] _ Deligne homology, Hodge-D-conjecture, and motives, In Beilinson's conjectures on special values of $L$-functions, 305-372, Perspect. Math., 4, Academic Press, Boston, MA, 1988.

[K] M.Kashiwara, A study of variation of mixed Hodge structure, Publ. RIMS. Kyoto Univ. 22 (1986), 991-1024.

[Me] Z.Mebkhout, Les formalisme des six opérations de Grothendieck pour les $D_{X}$-modules cohérents, Travaux en cours 35, Hermann: Paris 1989.

[M] D.Mumford, Rational equivalence of O-cycles on surfaces, J. Math. Kyoto Univ. 9 (1969), 195-204.

[Mur1] J.P.Murre, On a conjectural filtration on Chow groups of an algebraic variety, Indag. Math, New Series, 4 (1993), 177-201.

[Mur2] - On the motives of an algebraic surface, J. Reine. Angew. Math. 409 (1990), 190-204.

[R] M.Raynaud, Courbes sur une variété abélienne et points de torsion, Invent.Math. 71 (1983), 207-233.

[SaM1] M.Saito, Modules de Hodge polarisables, Publ. RIMS. Kyoto Univ. 24 (1988), 849-995.

[SaM2] - Mixed Hodge modules, Publ. RIMS. Kyoto Univ. 26 (1990), 221-333.

[SaM3] On the formalism of mixed sheaves, (RIMS preprint) .

[SaS1] S.Saito, Motives and filtration on Chow groups, Invent. Math. 125 (1996), 149-196.

[SaS2] - Motivic filtration on Chow groups, II. (to appear).

[SZ] J.Steenbrink and S.Zucker, Variation of mixed Hodge structure, Invent. math. 80 (1985) no.3, 489-542.

[AS] M.Asakura and S.Saito, Filtration on Chow groups and higher Abel-Jacobi maps, (preprint).

[A] M.Asakura, Arithmetic Hodge structure and higher Abel-Jacobi maps, (preprint).

Research Institute for Mathematics Sciences, Kyoto University, Oiwakecho, SakyoKU, КYото, 606-8502, JAPAN

E-mail address: asakura@kurims.kyoto-u.ac.jp 